\title{
Crosstalk between RPE cells and choroidal endothelial cells via the ANXA1/FPR2/SHP2/NLRP3 inflammasome/pyroptosis axis promotes choroidal neovascularization
}

\section{Manhui Zhu}

lixiang eye hospital of soochow university

\section{Ying Wang}

Suzhou Municipal Hospital

\section{Linling Zhu}

LIXIANG EYE HOSPITAL OF SOOCHOW UNIVERSITY

\section{Shu Du}

LIXIANG EYE HOSPITAL OF SOOCHOW UNIVERSITY

\section{Zhenzhen Wang}

LIXIANG EYE HOSPITAL OF SOOCHOW UNIVERSITY

\section{Yuting Zhang}

LIXIANG EYE HOSPITAL OF SOOCHOW UNIVERSITY

\section{Yang Guo}

LIXIANG EYE HOSPITAL OF SOOCHOW UNIVERSITY

\section{Yuanyuan Tu}

lixiang eye hospital of soochow university

\section{E Song ( $\nabla$ songe@suda.edu.cn )}

Soochow University https://orcid.org/0000-0003-4259-9739

\section{Research Article}

Keywords: Age-related macular degeneration (AMD), Choroidal neovascularization (CNV), Retinal pigment epithelial (RPE) cells, Choroidal endothelial cells (CECs), Annexin A1 (ANXA1), Nod-like receptor family, pyrin domain containing 3 (NLRP3) inflammasome

Posted Date: May 11th, 2021

DOl: https://doi.org/10.21203/rs.3.rs-497782/v1

License: (c) (i) This work is licensed under a Creative Commons Attribution 4.0 International License. Read Full License 
Version of Record: A version of this preprint was published at Inflammation on October 1st, 2021. See the published version at https://doi.org/10.1007/s10753-021-01555-3. 


\section{Abstract}

One type of age-related macular degeneration (AMD), neovascular (nAMD), characterized by choroidal neovascularization (CNV), accounts for the majority of the severe central vision impairment associated with AMD. Endothelial cells (ECs) in direct contact with retinal pigment epithelial (RPE) cells are more prone to the pathological angiogenesis involved in CNV. Herein, we investigated the effect of crosstalk between RPE cells and choroidal endothelial cells (CECs) via the ANXA1/FPR2/NLRP3 inflammasome/pyroptosis axis on the development of choroidal neovascularization (CNV) in vitro and in vivo. ANXA1 expression and secretion from ARPE-19 cells were upregulated by hypoxia. FPR2 expression, especially on the plasma membrane, in HCECs was upregulated under hypoxic conditions. ANXA1 secreted from ARPE-19 cells inhibited NLRP3 inflammasome activation and NLRP3 inflammasomemediated pyroptosis in HCECs by activating the FPR2/SHP2 axis. Moreover, ANXA1 secreted by ARPE-19 cells promoted behaviors of HCECs, including proliferation, migration and tube formation, by activating the FPR2/SHP2 axis and inhibiting NLRP3 inflammasome-mediated pyroptosis. Inhibiting the upregulated ANXA1/FPR2/SHP2/NLRP3 inflammasome/pyroptosis axis decreased the volume of CNV. Our data suggest that the crosstalk between RPE cells and CECs via the ANXA1/FPR2/NLRP3 inflammasome/pyroptosis axis promotes CNV. This finding could identify a potential target for the prevention and treatment of CNV.

\section{Introduction}

Age-related macular degeneration (AMD), a neurodegenerative disease, acts as is one of the leading causes of vision impairment in elderly individuals worldwide [15]. Advanced AMD is segmented into two types: neovascular AMD (nAMD) and nonneovascular AMD (aAMD). Among them, nAMD is characterized by choroidal neovascularization (CNV) that penetrates Bruch's membrane into the subretinal pigment epithelial space and the subretinal space, resulting in leakage, hemorrhage, edema, pigment epithelial detachment, and subretinal fibrosis [18], accounting for approximately $80 \%$ of the severe central vision impairment associated with AMD [26]. Recently, the first-line therapeutic strategy for nAMD is repeat intravitreal injection of anti-vascular endothelial growth factor (VEGF) agents, which can effectively delay the progression of nAMD. However, resistance to anti-VEGF drugs can occur from the beginning of treatment or be induced gradually. For example, the comparison of age-related macular degeneration treatments trials (CATT) reveals that despite monthly treatments with anti-VEGF agents for 2 years, $51.5 \%$ of patients receiving intravitreal ranibizumab (RAN) and $67.4 \%$ of patients that are treated with bevacizumab (BEV) exhibit evidence of persistent fluid as determined by time-domain optical coherence tomography (OCT) [7]. Anti-VEGF treatment resistance indicates that in addition to VEGF, other factors also contribute to the development of CNV.

Clinicopathological studies show that $50 \%$ of sight-threatening nAMD occurs when choroidal endothelial cells (CECs) are induced to migrate toward and make contact with the retinal pigment epithelium (RPE) and its extracellular matrix. After making contact with RPE, CECs can migrate across the RPE into the neurosensory retina, where CNV occurs [31]. VEGF 189 secreted by RPE cells facilitates the migration of 
CECs across the RPE [34]. Additionally, co-culture of endothelial cells (ECs) and RPE cells under conditions enabling direct EC-RPE cell contact enhances the proangiogenic potential of ECs under normoxic conditions to an extent similar to that induced by hypoxia, suggesting that ECs in direct contact with RPE cells might be more prone to the pathological angiogenesis involved in CNV [9].

Annexin A1 (ANXA1) is a major driver of inflammatory resolution. In vitro studies confirm that ANXA1 levels in RPE cells are upregulated after 60 and 120 min of infection with Toxoplasma gondii [21]. Using a false discovery rate of $20 \%$ as the threshold, the researchers identify 1,133 genes, including ANXA1 [log $(\mathrm{AMD} / \mathrm{cnt})=-0.41$ ], in GSE29801 [transcriptome data which is from macular and extramacular RPEchoroid tissue isolated from AMD patients with Rotterdam grades of 2a, 2b, or 3 and from individuals with no features of AMD (controls)], and these genes are dysregulated in macular RPE-choroid tissue from AMD patients compared with that of the controls [2]. Additionally, hypoxia induces ANXA1 expression in multiple cancers, such as colon cancer [23] and prostate cancer [4]. Therefore, we hypothesize that RPE cells release increased ANXA1 under hypoxic conditions during CNV.

Secreted ANXA1 exerts the inflammation resolution function by binding to its receptor formyl peptide receptor 2 (FPR2) [8], which is a G protein-coupled receptor (GPCR). Neither formyl peptide receptor 1 (FPR1) nor formyl peptide receptor 3 (FPR3) are affected by ANXA1, except FPR2 protein levels, which are increased in mouse laser-induced $15 \mathrm{~d}$ CNV lesions [5]. After ANXA1 binds to FPR2, ANXA1 interacts with $\mathrm{SH} 2$ domain-containing protein tyrosine phosphatase (SHP2), a ubiquitously existing non-receptor protein tyrosine phosphatase [6]. Inflammasomes are multimolecular signaling complexes that have crucial roles in host defense against various autoimmune and inflammatory disorders. Among the numerous types of inflammasomes that have been determined, the Nod-like receptor family pyrin domain containing 3 (NLRP3) inflammasome is the most extensively studied type owing to its mighty activation in response to multiple stimuli, including hypoxia [35]. Following activation, the NLRP3 inflammasome brings about caspase 1-dependent secretion of the proinflammatory cytokines interleukin-1 $\beta$ (IL-1 $\beta$ ) and interleukin-18 (IL-18), accompanied with gasdermin D (GSDMD)-assisted cell death named as pyroptosis. GSDMD contains an amino-terminal cell death domain (GSDMD ${ }^{\text {Nterm }}$ ), a central short linker region, and a carboxyterminal autoinhibition domain. Caspase 1 cleaves GSDMD, dislodging its carboxyl terminus and discharging it from intramolecular inhibition [28]. GSDMD ${ }^{\text {Nterm }}$ then binds to phosphatidylinositol phosphates and phosphatidylserine locating in the cell membrane inner leaflet, initiating pyroptosis [10]. SHP2 negatively modulates NLRP3 inflammasome activation in macrophages, consequently allevating NLRP3 inflammasome-mediated pyroptosis [14].

Herein, we investigated the effect of crosstalk between RPE cells and CECs via the ANXA1/FPR2/NLRP3 inflammasome/pyroptosis axis on CNV. Our data further clarify the crosstalk between RPE cells and CECs during CNV and hint potential targets for the more effective treatment of CNV.

\section{Materials And Methods}

\section{Cell culture and treatments}


The human RPE cell line ARPE-19 (CRL-2302, ATCC, USA) and human CECs (HCECs, CP-H092, Procell, China) were grown in Dulbecco's modified Eagle medium/Ham's F12 medium (DMEM/F-12; 8118247, Gibco, USA) supplemented with 10\% fetal bovine serum (FBS; 10099141, Gibco) and antibioticantimycotic solution (15240062, Gibco). ARPE-19 cells and HCECs cultured in $95 \%$ air and $5 \% \mathrm{CO}_{2}$ for 24 $\mathrm{h}$ were taken as the normal (normoxia) groups. Cells cultured in $1 \% \mathrm{O}_{2}, 5 \% \mathrm{CO}_{2}$ and $94 \% \mathrm{~N}_{2}$ in an oxygencontrolled chamber for $24 \mathrm{~h}$ were taken as the hypoxia groups. HCECs were treated with human recombinant ANXA1 protein (3770-AN, R\&D Systems, USA; $100 \mathrm{nM}$ for $24 \mathrm{~h}$ ), WRW4 (FPR2 antagonist; 2262, Tocris, USA; $10 \mu \mathrm{M}$ for 24 h), SHP099 (SHP2 inhibitor; S8278, Selleck, USA; $0.1 \mu \mathrm{M}$ for $24 \mathrm{~h}$ ), ARPE19 cell conditioned culture medium (CCM; hypoxic culture for $24 \mathrm{~h}$ ), ANXA1 neutralizing antibodies (ab46686, Abcam, USA; $1 \mu \mathrm{M}$ for $24 \mathrm{~h}$ ), adenosine triphosphate (ATP; NLRP3 inflammasome agonist; 10988537001, Roche, USA; 5 mM for 24 h) or a caspase-1 CRISPR activation plasmid (sc-417320-ACT, Santa Cruz Biotechnology, USA; $1 \mu \mathrm{g}$ for $24 \mathrm{~h}$ ).

\section{Western blot}

The cells were cultured to $70 \%$ confluence, and subjected to the designated treatments. The cells were lysed at $4^{\circ} \mathrm{C}$ using radioimmunoprecipitation assay (RIPA) lysis buffer (R0278, Sigma Aldrich, USA). Protein concentrations were determined using a BCA protein assay kit (A53225, Thermo Fisher Scientific, USA). Samples ( $80 \mu$ g protein) were resolved by $4-20 \%$ sodium dodecyl sulfate-polyacrylamide gel electrophoresis (SDS-PAGE; WXP81612BOX, Invitrogen, USA), transferred to polyvinylidene difluoride (PVDF) membranes (IPVH00010, Millipore, USA), and incubated with one of the following antibodies: ANXA1 (21990-1-AP, Proteintech, USA), FPR2 (PA5-75750, Invitrogen), p-SHP2 (Tyr542; \#3751, Cell Signaling Technology), SHP2 (\#3752, Cell Signaling Technology), ASC (67494-1-lg, Proteintech), NLRP3 (19771-1-AP, Proteintech), IL-1 $\beta$ (including pro-IL-1 $\beta$ and cleaved-IL-1 $\beta$; AF-401, R\&D Systems), N-GSDMD (ab215203, Abcam), GSDMD (20770-1-AP, Proteintech) and caspase-1 (22915-1-AP, Proteintech). The membranes were washed three times with phosphate-buffered saline with Tween detergent (PBST) and incubated with horseradish peroxidase (HRP)-conjugated goat-anti-rabbit (SA00001-2, Proteintech; 1:5000) or HRP-conjugated goat-anti-mouse antibodies (SA00001-1, Proteintech; 1:5000) at room temperature for 2 h. GAPDH (600004-1-IG, Proteintech; 1:50000) and caveolin 1 (Cav1; 16447-1-AP, Proteintech) were used as loading controls. Unless otherwise indicated, the dilution for each antibody was 1:1000. Following an additional 10 min of washing with PBST, the protein bands were visualized using an electrochemiluminescence (ECL) assay kit (JP001B250, CLINX, China). The density of each band was measured using Image J software. The expression level of each target protein was normalized to the GAPDH or Cav1 protein expression level and compared with that of the normal group, which was assigned a value of 1 .

\section{Enzyme-linked immunosorbent assay (ELISA)}

The levels of ANXA1 were measured using a human ANXA1 ELISA kit (NBP2-60538, Novus Biologicals, USA) according to the manufacturer's instructions. The detection range of the kit was $0.313-20 \mathrm{ng} / \mathrm{ml}$. 
The absorbance was measured using a microplate reader (Bio-Rad Laboratories, USA) at a wavelength of $450 \mathrm{~nm}$ and a correction at $655 \mathrm{~nm}$.

\section{Plasma membrane and cytosol isolation}

Plasma membrane and cytosolic fractions were isolated from HCECs using a plasma membrane protein extraction kit (ab65400, Abcam, USA).

\section{Terminal deoxynucleotidyl transferase dUTP nick-end labeling (TUNEL) assay}

HCECs were seeded on glass coverslips in 24-well plates. After different treatments for $24 \mathrm{~h}$, the TUNEL assay was done using a in situ cell death detection kit (11684795910, Roche), and the cells were observed under a TCS-SP2 confocal microscope (Leica Microsystems, Germany).

\section{5-Ethynyl-2'-deoxyuridine (EdU) incorporation assay}

Cell proliferation was analyzed using the EdU Apollo 488 assay kit (C10310-1, RiboBio, China). The images were viewed using a confocal microscope at $100 \times$ magnification. The number of stained nuclei was counted and used to determine the percentage of the total number of nuclei in each image.

\section{Wound healing assay}

Confluent monolayers of HCECs in 6-well plates were scratched with pipet tips, leading to one acellular 0.5-mm-wide lane per well. After the cells were washed twice, the HCECs were exposed to different treatments. Photographs were taken after $24 \mathrm{~h}$ of incubation. The amount of migration was determined by ImageJ software.

\section{Tube formation assay}

A 24-well culture plate was coated with $100 \mu \mathrm{l}$ of $10 \mathrm{mg} / \mathrm{ml}$ precooled growth factor-reduced Matrigel (354230, Corning, USA). HCECs were seeded on Matrigel-coated plates at concentrations of $5 \times 10^{4}$ cells per well and exposed to different treatments. After $24 \mathrm{~h}$ of culture, images were acquired using an optical microscope ( $\times 100$ magnification). The average length of the tube branches in four areas of each sample was assessed under an inverted light microscope.

\section{Mouse laser-induced CNV model}

After anesthesia and pupil dilation, male C57BL/6J mice (purchased from the Laboratory Animal Center of Soochow University) (aged 10 weeks) were subjected to laser photocoagulation using a PASCAL diode ophthalmic laser system (neodymium-doped yttrium aluminum garnet [Nd:YAG], $532 \mathrm{~nm}$; Topcon Medical Laser Systems, Santa Clara, USA) with the following parameters including $200 \mathrm{~mm}$ spot size, $0.02 \mathrm{~s}$ duration, and $100 \mathrm{~mW}$ power. Four laser spots were made around the optic nerve heads of both eyes in each mouse to induce CNV. The rupture of Bruch's membrane was verified by observing a bubble at each 
laser spot. Approval was obtained from the Animal Research Ethics Committee of Soochow University in agreement with the Chinese National Standard.

The mice were allocated into the normal, CNV $7 d$, CNV $7 d+$ PBS, CNV $7 d+$ ANXA1 neutralizing antibody (intravitreal injection of $2 \mu \mathrm{l}$ of $50 \mu \mathrm{g} / \mathrm{ml}$ antibody on day 3), CNV $7 \mathrm{~d}+$ WRW4 (intraperitoneal injection; 1 $\mu \mathrm{g} / \mathrm{kg} / \mathrm{d}$ from day 0 to day 6), CNV $7 \mathrm{~d}+\mathrm{SHP099}$ (oral gavage, $50 \mathrm{mg} / \mathrm{kg} / \mathrm{d}$ from day 0 to day 6 ), CNV $7 \mathrm{~d}$ + ATP (intravitreal injection; $5 \mathrm{mM}$ on day 3), and CNV + caspase-1 plasmid (intravitreal injection; $1 \mu \mathrm{g}$ on day 3) groups. There were five mice in each group. Three mice were excluded due to hemorrhage at the site of laser administration.

\section{Immunohistochemical analysis of RPE-choroid cryosections}

Immunohistochemical analysis of ANXA1, FPR2, p-SHP2, NLRP3 or caspase-1 was performed on flattened retina-RPE-choroid complexes $7 \mathrm{~d}$ after laser exposure as previously described [32]. The antibodies used immunohistochemical analysis included anti-ANXA1 (PA5-27315, Invitrogen), anti-FPR2 (NLS1878, Novus Biologicals, USA), anti-p-SHP2 (STJ90741, St. John's Laboratory, USA), anti-NLRP3 (MA5-32255, Invitrogen), and anti-caspase-1 (MA5-32909, Invitrogen).

\section{Immunofluorescence analysis of mouse choroidal flat mounts}

The mouse choroidal flat mounts were prepared as previously described [3]. The antibodies and reagents used included Alexa Fluor ${ }^{\text {TM }}$ 488-conjugated anti-isolectin B4 (IB4) from Griffonia simplicifolia (I21411, Invitrogen), Alexa Fluor ${ }^{\text {TM }}$ 594-conjugated anti-collagen IV (ITT5767, G-Biosciences, USA) and 4', 6diamidino-2-phenylindole (DAPI; D9542, Sigma Aldrich).

\section{Statistical analysis}

All results are represented as the means \pm SEM. Statistical significance between groups was evaluated with Student's unpaired t-tests (two-tailed). A value of $P<0.05$ was considered to be statistically significant.

\section{Results}

\section{ANXA1 expression and secretion from ARPE-19 cells are upregulated by hypoxia.}

Ocular Toxoplasma gondii infection contributes to a significant increase in ANXA1 expression in the mouse RPE at $48 \mathrm{~h}$ and $72 \mathrm{~h}$ [21]. First, we measured ANXA1 protein levels in hypoxia-exposed ARPE-19 cells at different time points and found that ANXA1 protein levels increased in ARPE-19 cells in response to hypoxic conditions at $16 \mathrm{~h}$, peaked at $24 \mathrm{~h}$, and then declined (Fig. 1A and 1B). Moreover, ANXA1 secreted by ARPE-19 cells under hypoxic conditions was measured by ELISA and showed a similar tendency (Fig. 1C). These data suggested that ANXA1 expression and secretion from ARPE-19 cells were upregulated by hypoxia. 
FPR2 expression, especially on the plasma membrane, in HCECs is upregulated under hypoxic conditions.

ANXA1 binds to FPR2 to exert an anti-inflammatory effect [19], and FPR2 is expressed in multiple types of ECs, including human retinal microvascular ECs [27]. Based on previous studies, we hypothesize that HCECs express FPR2, which binds to ANXA1 produced by RPE cells under hypoxic conditions. As expected, FPR2 protein levels in normal HCECs were low. After hypoxia exposure, FPR2 protein levels increased at $16 \mathrm{~h}$, reaching a peak at $24 \mathrm{~h}$ (Fig. 2A and 2B). Additionally, following culture under hypoxic conditions for $24 \mathrm{~h}$, HCEC FPR2 expression on the plasma membrane but not in the cytosol increased (Fig. 2C). These results suggested that FPR2 expression, especially on the plasma membrane of HCECs, was upregulated by hypoxia.

\section{ANXA1 secreted by ARPE-19 cells inhibits NLRP3 inflammasome activation in HCECs by activating the FPR2/SHP2 axis.}

The ANXA1 tripeptide suppresses NLRP3 inflammasome activation, thus mitigating microglial activation and hippocampal-dependent memory deficits [13]. In addition, FPR2 promotes SHP2 activation [22]. Therefore, whether ANXA1 interacted with FPR2 to activate SHP2 and subsequently inhibited NLRP3 inflammasome activation was examined. Hypoxia induced SHP2 phosphorylation compared to that of normal (normoxic) conditions. Human ANXA1 recombinant protein also induced the phosphorylation of SHP2, while the FPR2 antagonist WRW4 and SHP2 inhibitor SHP099 impaired the positive effect of ANXA1. CCM from ARPE-19 cells cultured under hypoxic conditions for $24 \mathrm{~h}$ also enhanced the phosphorylation of SHP2, while the ANXA1 neutralizing antibody WRW4 or SHP099 inhibited the effect of CCM. Except in the normoxia and hypoxia groups, the NLRP3 inflammasome-associated molecules ASC, NLRP3 and IL-1 $\beta$, showed the opposite tendencies of $p-S H P$ (Fig. 3A-3E). Pro-IL-1 $\beta$ showed few changes in each group. These data suggested that ANXA1 secreted by ARPE-19 cells inhibited NLRP3 inflammasome activation in HCECs by activating the FPR2/SHP2 axis.

\section{ANXA1 secreted by ARPE-19 cells inhibits NLRP3 inflammasome-mediated pyroptosis in HCECs by activating the FPR2/SHP2 axis.}

Next, the effect of the ANXA1/FPR2/SHP2 axis on NLRP3 inflammasome-mediated pyroptosis in HCECs was examined. N-GSDMD and cleaved caspase-1 were upregulated in the hypoxia group compared to the normoxia group. The administration of ANXA1 protein decreased N-GSDMD and cleaved caspase-1 protein levels, while WRW4, SHP099, the NLRP3 inflammasome agonist ATP or caspase-1 activation plasmid reversed the effect of ANXA1. Similarly, CCM downregulated N-GSDMD and cleaved caspase-1 protein levels, and WRW4, ANXA1 neutralizing antibody, SHP099, ATP or caspase-1 activation plasmid inhibited the effects of CCM (Fig. 4A-4C). Moreover, the ratio of TUNEL-positive HCECs showed a similar tendency as the N-GSDMD and cleaved caspase-1 protein levels (Fig. 4D-4E). Therefore, these results suggested that ANXA1 secreted by ARPE-19 cells inhibits NLRP3 inflammasome-mediated pyroptosis in HCECs by activating the FPR2/SHP2 axis. 
ANXA1 secreted by ARPE-19 cells promotes HCECs proliferation, migration and tube formation by activating the FPR2/SHP2 axis and inhibiting NLRP3 inflammasome-mediated pyroptosis.

Then, we examined the behaviors of HCECs associated with ANXA1/FPR2/SHP2-regulated pyroptosis. HCECs proliferation, migration and tube formation was increased in the hypoxia group compared to the normoxia group. The administration of ANXA1 protein increased the proliferation, migration and tube formation of HCECs, while WRW4, SHP099, ATP or caspase-1 activation plasmid blocked the effects of ANXA1. Furthermore, CCM enhanced the proliferation, migration and tube formation of HCECs, while ANXA1 neutralizing antibody, WRW4, SHP099, ATP or caspase-1 activation plasmid inhibited the effects of CCM (Fig. 5A-5F). These data suggested that ANXA1 secreted by ARPE-19 cells promoted the proliferation, migration and tube formation of HCECs by activating the FPR2/SHP2 axis and inhibiting NLRP3 inflammasome-mediated pyroptosis.

\section{The ANXA1/FPR2/SHP2/NLRP3 inflammasome/pyroptosis axis is enhanced in mouse laser-induced CNV.}

To further examine the expressions of ANXA1/FPR2/SHP2/NLRP3 inflammasome/pyroptosis axis factors, a mouse laser-induced CNV model was established. Immunohistochemical analysis of mouse retinal-RPE-choroid tissues showed that ANXA1 (Fig. 6A), FPR2 (Fig. 6B), p-SHP2 (Fig. 6C), NLRP3 (Fig. 6D) and caspase-1 (Fig. 6E) increased in the CNV $7 \mathrm{~d}$ group compared to the normal group, suggesting that the ANXA1/FPR2/SHP2/NLRP3 inflammasome/pyroptosis axis increased in laser-induced CNV mice.

\section{Inhibition of the ANXA1/FPR2/SHP2/NLRP3 inflammasome/pyroptosis axis decreases the volume of CNV.}

Finally, we examined the effect of the ANXA1/FPR2/SHP2/NLRP3 inflammasome/pyroptosis axis on the volume of CNV and found that blocking the axis decreased the volume of laser-induced CNV in mice (Fig. 7A and 7B). The mechanism of the ANXA1/FPR2/SHP2/NLRP3 inflammasome/pyroptosis axis in CNV is shown in Fig. 7C.

\section{Discussion}

First, we found that ANXA1 expression and secretion from hypoxia-exposed ARPE-19 cells were higher than those in normal (hypoxia for $0 \mathrm{~h}$ ) cells. A previous study reveals that ANXA1 is upregulated under low oxygen conditions by hypoxia-inducible factor (HIF-1) overexpression and not by binding to a hypoxia-responsive element (HRE; -430 5'-CACCT-3' -426) in the ANXA1 promoter [17]. However, another study shows that the loss of HIF-1a can retain tumor metabolism and proliferation by boosting ANXA1, and these proteins are expressed in a mutually exclusive way in 37 gastric cancer (GC) cell lines. ANXA1 is an crucial protein in the downstream pathway regulated by HIF-1a under hypoxic conditions [25]. Whether ANXA1 is upregulated by HIF-1a at the transcriptional level requires further examination. 
ANXA1 promotes cardiac macrophages to release high level of VEGF-A (VEGF is commonly refers to VEGF-A), hence triggering cardiac neovascularization and repair [12]. Moreover, ANXA1 promotes EC migration and angiogenesis [24]. The proangiogenic effects of ANXA1 can be explained by the signal amplifier role of ANXA1 to boost the release of second messengers that impact cellular proliferation and migration [30]. In our study, we found that ANXA1 promoted proliferation, migration, and tube formation of HCECs by downregulating downstream NLRP3 inflammasome activation and pyroptosis, indicating that the proangiogenic effects of ANXA1 are associated with inhibiting inflammation.

Next, we found that FPR2 expression, especially on the plasma membrane, in CECs was upregulated by hypoxia. The transcription of FPR2 is precisely modulated by numerous transcription factors, including octamer-binding protein 1 (OCT1) [33], specificity protein 1 (SP1) [29], and signal transducer and activator of transcription 3 (STAT3) [1]. Evidence shows that the phosphorylation of STAT3 is pivotal for the induction of FPR2 responsive to double-stranded RNA [1]. In addition, hypoxia-induced STAT3 activation facilitates angiogenesis and glioblastoma cell migration [16]. Thus, we hypothesize that hypoxia-induced phosphorylation of STAT3 promotes the transcription of FPR2 in HCECs.

NLRP3 inflammasome activation in non-RPE cells, but not in RPE cells, promotes CNV in an nAMD mouse model [20]. However, NNLRP3 inflammasome activation occurs in macrophages and results in IL-18 activation, which inhibits laser-induced CNV [11]. The difference in these conclusions may be derived from the different animal models used in the two studies: the former uses a genetic model of nAMD Vegfahyper mice, and the latter uses a laser-induced CNV mouse model. In our study, we find that SHP2 inhibits NLRP3 inflammasome activation in HCECs to exacerbate the formation of laser-induced CNV in mice, while the SHP2 inhibitor SHP099 alleviates CNV.

In summary, we elucidated a mechanism by which the crosstalk between RPE cells and CECs via the ANXA1/FPR2/SHP2/NLRP3 inflammasome/pyroptosis axis promotes CNV. There were several limitations in our study worth mentioning, such as the lack of primary cell experiments and the absence of FPR1 and FPR3 detection in HCECs. However, our identification of crosstalk may facilitate the manipulation of FPR2 expression to develop therapeutics for CNV.

\section{Declarations}

\section{AUTHOR'S CONTRIBUTION}

M. H. Z and E. S. designed the research and wrote the manuscript; M. H. Z, Y. W., L. L. Z., S. D., Z. Z. W., Y. Y. Z., Y. G. and Y. Y. T. performed the experiments; Y. Y. T. and Y. W. collected and analyzed the data.

\section{FUNDING INFORMATION}

The study was supported by the Suzhou Science and Technology Bureau (No. SYS2018005), the National Natural Science Foundation of China (No. 81770906), the Jiangsu Provincial Natural Science Foundation 
Project (Nos. BK20200209 \& BK BK20191177), the Suzhou Commission of Health and Family Planning (No. KJXW2018076) and the Project of Suzhou Lixiang Eye Hospital (No. SLKY2020120).

\section{DATA AVAILABILITY}

Not applicable

\section{Ethical Approval}

All mice studies were conducted according to protocols approved by the Animal Ethics Committee of Soochow University.

\section{Consent for Publication}

All authors have reviewed the manuscript and have given consent for publication.

\section{Conflicts of interest}

The authors declare that there are no conflicts of interest.

\section{References}

1. Ampomah, P. B., L. A. Moraes, H. M. Lukman, and L. H. K. Lim. 2018. Formyl peptide receptor 2 is regulated by RNA mimics and viruses through an IFN-beta-STAT3-dependent pathway. FASEB J 32 (3):1468-1478. doi:10.1096/fj.201700584RR.

2. Ashikawa, Y., Y. Nishimura, S. Okabe, Y. Sato, M. Yuge, T. Tada, H. Miyao et al. . 2017. Potential protective function of the sterol regulatory element binding factor 1 -fatty acid desaturase $1 / 2$ axis in early-stage age-related macular degeneration. Heliyon 3 (3):e00266. doi:10.1016/j.heliyon.2017.e00266.

3. Balser, C., A. Wolf, M. Herb, and T. Langmann. 2019. Co-inhibition of PGF and VEGF blocks their expression in mononuclear phagocytes and limits neovascularization and leakage in the murine retina. J Neuroinflammation 16 (1):26. doi:10.1186/s12974-019-1419-2.

4. Bizzarro, V., R. Belvedere, V. Migliaro, E. Romano, L. Parente, and A. Petrella. 2017. Hypoxia regulates ANXA1 expression to support prostate cancer cell invasion and aggressiveness. Cell Adh Migr 11 (3):247-260. doi:10.1080/19336918.2016.1259056.

5. Cammalleri, M., M. Dal Monte, F. Locri, L. Lista, M. Aronsson, A. Kvanta, D. Rusciano et al. . 2016. The Urokinase Receptor-Derived Peptide UPARANT Mitigates Angiogenesis in a Mouse Model of LaserInduced Choroidal Neovascularization. Invest Ophthalmol Vis Sci 57 (6):2600-2611. doi:10.1167/iovs.15-18758.

6. Colavita, I., N. Esposito, C. Quintarelli, E. Nigro, F. Pane, M. Ruoppolo, and F. Salvatore. 2013. Identification of Annexin A1 interacting proteins in chronic myeloid leukemia KCL22 cells. Proteomics 13 (16):2414-2418. doi:10.1002/pmic.201200444. 
7. Comparison of Age-related Macular Degeneration Treatments Trials Research, Group, D. F. Martin, M. G. Maguire, S. L. Fine, G. S. Ying, G. J. Jaffe, J. E. Grunwald, C. Toth, M. Redford, and F. L. Ferris, 3rd. 2012. Ranibizumab and bevacizumab for treatment of neovascular age-related macular degeneration: two-year results. Ophthalmology 119 (7):1388-1398. doi:10.1016/j.ophtha.2012.03.053.

8. Cooray, S. N., T. Gobbetti, T. Montero-Melendez, S. McArthur, D. Thompson, A. J. Clark, R. J. Flower, and M. Perretti. 2013. Ligand-specific conformational change of the G-protein-coupled receptor ALX/FPR2 determines proresolving functional responses. Proc Natl Acad Sci U S A 110 (45):1823218237. doi:10.1073/pnas.1308253110.

9. Dardik, R., T. Livnat, Y. Nisgav, and D. Weinberger. 2010. Enhancement of angiogenic potential of endothelial cells by contact with retinal pigment epithelial cells in a model simulating pathological conditions. Invest Ophthalmol Vis Sci 51 (12):6188-6195. doi:10.1167/iovs.09-5095.

10. Ding, J., K. Wang, W. Liu, Y. She, Q. Sun, J. Shi, H. Sun, D. C. Wang, and F. Shao. 2016. Pore-forming activity and structural autoinhibition of the gasdermin family. Nature 535 (7610):111-116. doi:10.1038/nature18590.

11. Doyle, S. L., M. Campbell, E. Ozaki, R. G. Salomon, A. Mori, P. F. Kenna, G. J. Farrar et al. . 2012. NLRP3 has a protective role in age-related macular degeneration through the induction of IL-18 by drusen components. Nat Med 18 (5):791-798. doi:10.1038/nm.2717.

12. Ferraro, B., G. Leoni, R. Hinkel, S. Ormanns, N. Paulin, A. Ortega-Gomez, J. R. Viola et al. . 2019. ProAngiogenic Macrophage Phenotype to Promote Myocardial Repair. J Am Coll Cardiol 73 (23):29903002. doi:10.1016/j.jacc.2019.03.503.

13. Galvao, I., R. V. H. de Carvalho, J. P. Vago, A. L. N. Silva, T. G. Carvalho, M. M. Antunes, F. M. Ribeiro et al. . 2020. The role of annexin A1 in the modulation of the NLRP3 inflammasome. Immunology 160 (1):78-89. doi:10.1111/imm.13184.

14. Guo, W., W. Liu, Z. Chen, Y. Gu, S. Peng, L. Shen, Y. Shen et al. . 2017. Tyrosine phosphatase SHP2 negatively regulates NLRP3 inflammasome activation via ANT1-dependent mitochondrial homeostasis. Nat Commun 8 (1):2168. doi:10.1038/s41467-017-02351-0.

15. Jin, G., M. Zou, A. Chen, Y. Zhang, C. A. Young, S. B. Wang, and D. Zheng. 2019. Prevalence of agerelated macular degeneration in Chinese populations worldwide: A systematic review and metaanalysis. Clin Exp Ophthalmol 47 (8):1019-1027. doi:10.1111/ceo.13580.

16. Kang, S. H., M. O. Yu, K. J. Park, S. G. Chi, D. H. Park, and Y. G. Chung. 2010. Activated STAT3 regulates hypoxia-induced angiogenesis and cell migration in human glioblastoma. Neurosurgery 67 (5):1386-1395; discussion 1395. doi:10.1227/NEU.0b013e3181f1c0cd.

17. Liao, S. H., X. Y. Zhao, Y. H. Han, J. Zhang, L. S. Wang, L. Xia, K. W. Zhao, Y. Zheng, M. Guo, and G. Q. Chen. 2009. Proteomics-based identification of two novel direct targets of hypoxia-inducible factor-1 and their potential roles in migration/invasion of cancer cells. Proteomics 9 (15):3901-3912. doi:10.1002/pmic.200800922. 
18. Lim, L. S., P. Mitchell, J. M. Seddon, F. G. Holz, and T. Y. Wong. 2012. Age-related macular degeneration. Lancet 379 (9827):1728-1738. doi:10.1016/S0140-6736(12)60282-7.

19. Machado, M. G., L. P. Tavares, G. V. S. Souza, C. M. Queiroz-Junior, F. R. Ascencao, M. E. Lopes, C. C. Garcia et al. . 2020. The Annexin A1/FPR2 pathway controls the inflammatory response and bacterial dissemination in experimental pneumococcal pneumonia. FASEB J 34 (2):2749-2764. doi:10.1096/fj.201902172R.

20. Malsy, J., A. C. Alvarado, J. O. Lamontagne, K. Strittmatter, and A. G. Marneros. 2020. Distinct effects of complement and of NLRP3- and non-NLRP3 inflammasomes for choroidal neovascularization. Elife 9. doi:10.7554/eLife.60194.

21. Mimura, K. K., R. C. Tedesco, K. S. Calabrese, C. D. Gil, and S. M. Oliani. 2012. The involvement of anti-inflammatory protein, annexin A1, in ocular toxoplasmosis. Mol Vis 18:1583-1593.

22. Mitchell, D., S. J. O'Meara, A. Gaffney, J. K. Crean, B. T. Kinsella, and C. Godson. 2007. The Lipoxin A4 receptor is coupled to SHP-2 activation: implications for regulation of receptor tyrosine kinases. $J$ Biol Chem 282 (21):15606-15618. doi:10.1074/jbc.M611004200.

23. Onozawa, H., M. Saito, K. Saito, Y. Kanke, Y. Watanabe, S. Hayase, W. Sakamoto et al. . 2017. Annexin A1 is involved in resistance to 5-FU in colon cancer cells. Oncol Rep 37 (1):235-240. doi:10.3892/or.2016.5234.

24. Pin, A. L., F. Houle, P. Fournier, M. Guillonneau, E. R. Paquet, M. J. Simard, I. Royal, and J. Huot. 2012. Annexin-1-mediated endothelial cell migration and angiogenesis are regulated by vascular endothelial growth factor (VEGF)-induced inhibition of miR-196a expression. J Biol Chem 287 (36):30541-30551. doi:10.1074/jbc.M112.393561.

25. Rohwer, N., F. Bindel, C. Grimm, S. J. Lin, J. Wappler, B. Klinger, N. Bluthgen et al. . 2016. Annexin A1 sustains tumor metabolism and cellular proliferation upon stable loss of HIF1A. Oncotarget 7 (6):6693-6710. doi:10.18632/oncotarget.6793.

26. Rosenfeld, P. J., D. M. Brown, J. S. Heier, D. S. Boyer, P. K. Kaiser, C. Y. Chung, R. Y. Kim, and Marina Study Group. 2006. Ranibizumab for neovascular age-related macular degeneration. N Engl J Med 355 (14):1419-1431. doi:10.1056/NEJMoa054481.

27. Shi, H., T. W. Carion, Y. Jiang, J. J. Steinle, and E. A. Berger. 2016. VIP protects human retinal microvascular endothelial cells against high glucose-induced increases in TNF-alpha and enhances RvD1. Prostaglandins Other Lipid Mediat 123:28-32. doi:10.1016/j.prostaglandins.2016.03.001.

28. Shi, J., Y. Zhao, K. Wang, X. Shi, Y. Wang, H. Huang, Y. Zhuang, T. Cai, F. Wang, and F. Shao. 2015. Cleavage of GSDMD by inflammatory caspases determines pyroptotic cell death. Nature 526 (7575):660-665. doi:10.1038/nature15514.

29. Simiele, F., A. Recchiuti, D. Mattoscio, A. De Luca, E. Cianci, S. Franchi, V. Gatta et al. . 2012. Transcriptional regulation of the human FPR2/ALX gene: evidence of a heritable genetic variant that impairs promoter activity. FASEB J 26 (3):1323-1333. doi:10.1096/fj.11-198069.

30. Skouteris, G. G., and C. H. Schroder. 1996. The hepatocyte growth factor receptor kinase-mediated phosphorylation of lipocortin-1 transduces the proliferating signal of the hepatocyte growth factor. $J$ 
Biol Chem 271 (44):27266-27273. doi:10.1074/jbc.271.44.27266.

31. Stevens, T. S., N. M. Bressler, M. G. Maguire, S. B. Bressler, S. L. Fine, J. Alexander, D. A. Phillips, R. R. Margherio, P. L. Murphy, and A. P. Schachat. 1997. Occult choroidal neovascularization in age-related macular degeneration. A natural history study. Arch Ophthalmol 115 (3):345-350.

doi:10.1001/archopht.1997.01100150347006.

32. Valdes-Sanchez, L., S. M. Calado, B. de la Cerda, A. Aramburu, A. B. Garcia-Delgado, S. Massalini, A. Montero-Sanchez et al. . 2019. Retinal pigment epithelium degeneration caused by aggregation of PRPF31 and the role of HSP70 family of proteins. Mol Med 26 (1):1. doi:10.1186/s10020-019-0124$\mathrm{Z}$.

33. Waechter, V., M. Schmid, M. Herova, A. Weber, V. Gunther, J. Marti-Jaun, S. Wust, M. Rosinger, C. Gemperle, and M. Hersberger. 2012. Characterization of the promoter and the transcriptional regulation of the lipoxin A4 receptor (FPR2/ALX) gene in human monocytes and macrophages. $J$ Immunol 188 (4):1856-1867. doi:10.4049/jimmunol.1101788.

34. Wang, H., P. Geisen, E. S. Wittchen, B. King, K. Burridge, P. A. D'Amore, and M. E. Hartnett. 2011. The role of RPE cell-associated VEGF(1)(8)(9) in choroidal endothelial cell transmigration across the RPE. Invest Ophthalmol Vis Sci 52 (1):570-578. doi:10.1167/iovs.10-5595.

35. Watanabe, S., F. Usui-Kawanishi, T. Karasawa, H. Kimura, R. Kamata, T. Komada, Y. Inoue, N. Mise, T. Kasahara, and M. Takahashi. 2020. Glucose regulates hypoxia-induced NLRP3 inflammasome activation in macrophages. J Cell Physiol 235 (10):7554-7566. doi:10.1002/jcp.29659.

\section{Figures}

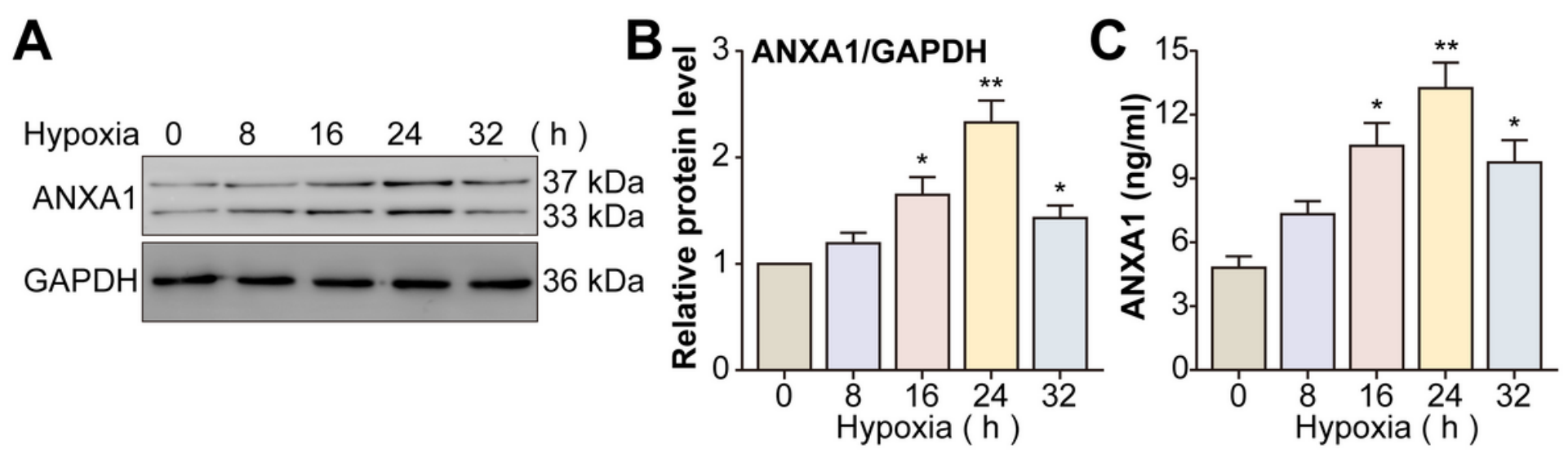

\section{Figure 1}

ANXA1 expression and secretion from ARPE-19 cells are upregulated by hypoxia. ARPE-19 cells were grown in a hypoxic environment for $0 \mathrm{~h}, 8 \mathrm{~h}, 16 \mathrm{~h}, 24 \mathrm{~h}$ or $32 \mathrm{~h}$. (A) ANXA1 protein level in ARPE-19 cells was measured by Western blot. GAPDH was used as the loading control. (B) The relative protein level of ANXA1 was analyzed. (C) The protein level of ANXA1 in ARPE-19 cell culture supernatant was measured by ELISA. In Fig. $1 \mathrm{~B}$ and $1 \mathrm{C},{ }^{*} \mathrm{P}<0.05$ and ${ }^{* *} \mathrm{P}<0.01$ versus the 0 h hypoxia group. $\mathrm{n}=4$ /each group. 
A

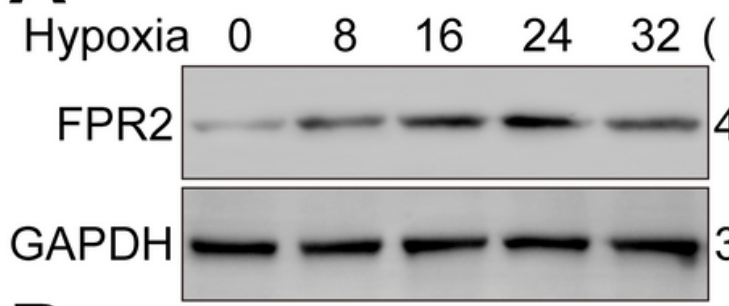

B

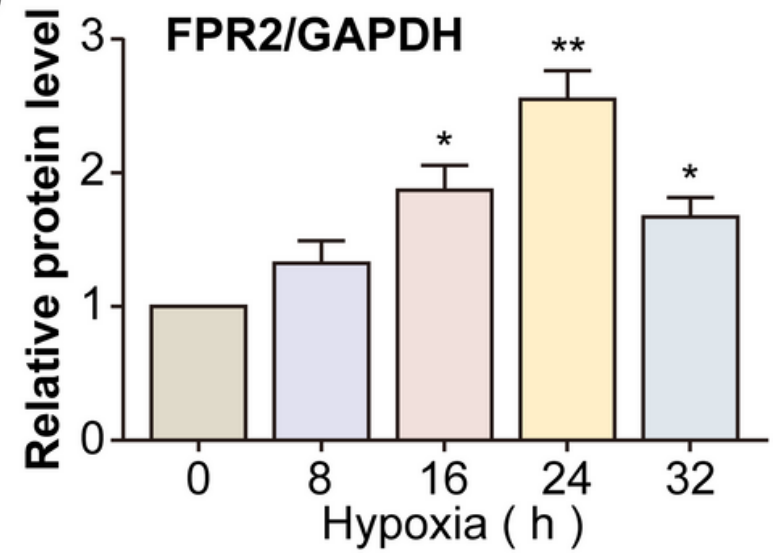
$40 \mathrm{kDa}$ $36 \mathrm{kDa}$ c

\section{Normal Hypoxia}

西

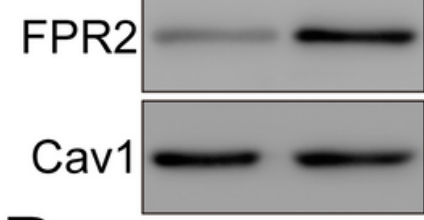

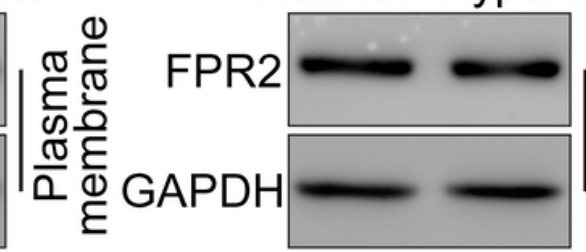

Normal Hypoxia

D |

Figure 2

FPR2 expression, especially on the plasma membrane, in HCECs is upregulated by hypoxia. HCECs were cultured under hypoxic conditions for $0 \mathrm{~h}, 8 \mathrm{~h}, 16 \mathrm{~h}, 24 \mathrm{~h}$ or $32 \mathrm{~h}$. (A) FPR2 protein level in HCECs was measured by Western blot. GAPDH was used as the loading control. (B) The relative protein level of FPR2 was analyzed. ${ }^{*} \mathrm{P}<0.05$ and ${ }^{*} \mathrm{P}<0.01$ versus the $0 \mathrm{~h}$ hypoxia group. (C) Following normoxic or hypoxic culture for $24 \mathrm{~h}$, the plasma membrane and cytosolic fractions of HCECs were isolated for analysis. Lysates were prepared, and Western blot was used to measure FPR2 protein levels. (D) The relative protein level of FPR2 in the plasma membrane and cytosol was analyzed. ${ }^{*} \mathrm{P}<0.01$ versus the normal group. The ns indicated no significance. $n=4$ /each group. 


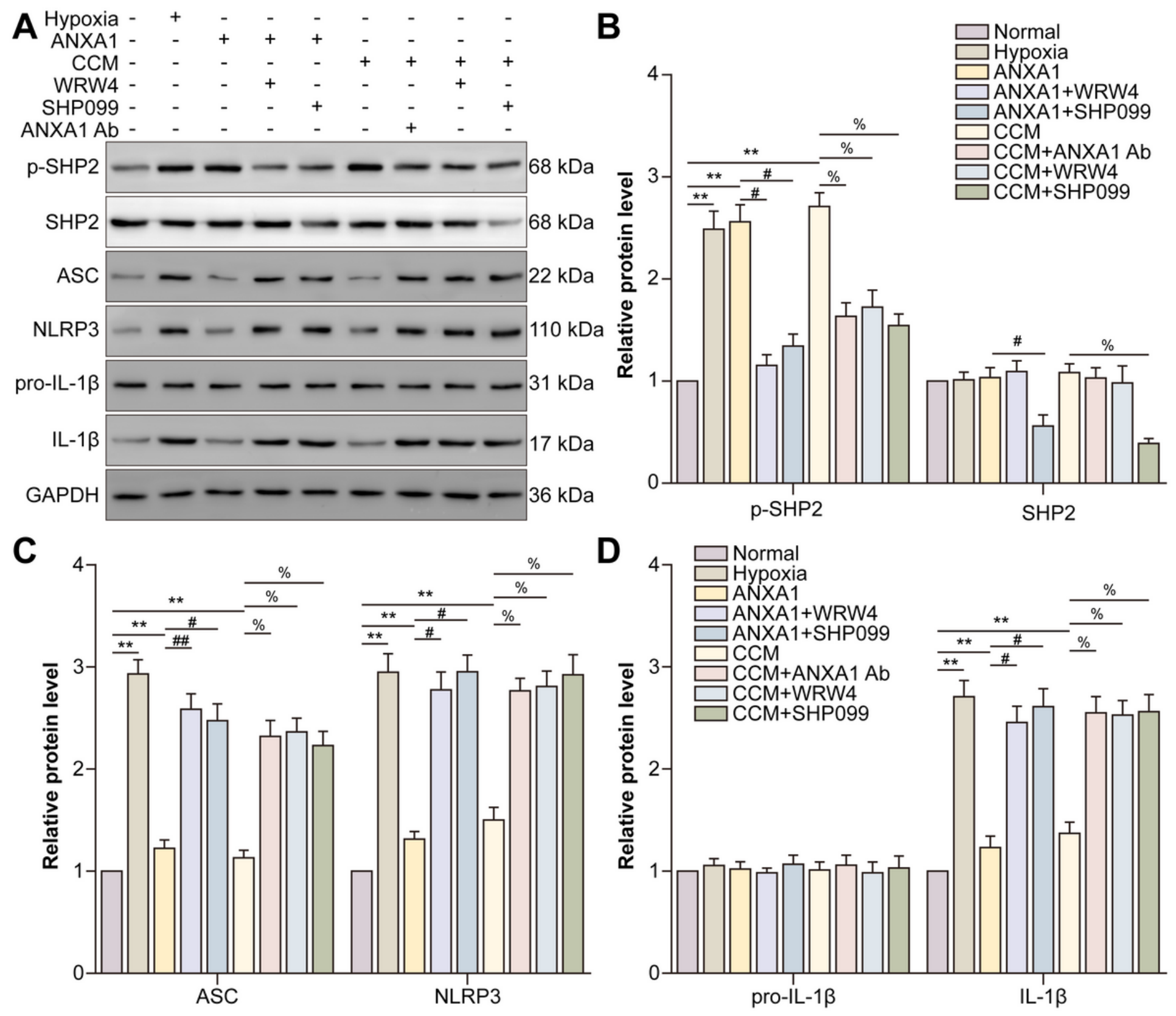

Figure 3

ANXA1 secreted by ARPE-19 cells inhibits NLRP3 inflammasome activation in HCECs by activating the FPR2/SHP2 axis. HCECs were allocated into the normal, hypoxia, human recombinant ANXA1 protein, ANXA1 + WRW4, ANXA1 + SHP099, ARPE-19 cell conditioned culture medium (CCM), CCM + ANXA1 neutralizing antibody, CCM + WRW4, and CCM + SHP099 groups. (A) The protein levels of NLRP3 inflammasome-associated molecules, including p-SHP, SHP2, ASC, NLRP3, pro-IL-1 $\beta$ and IL-1 $\beta$, were measured by Western blot. (B-D) The relative protein level of each molecule was analyzed. ${ }^{*} \mathrm{P}<0.01$ versus the normal group. \#P $<0.05$ and \#\#P $<0.01$ versus the ANXA1 group. $\% \mathrm{P}<0.05$ versus the CCM group. $n=4$ /each group. 
A

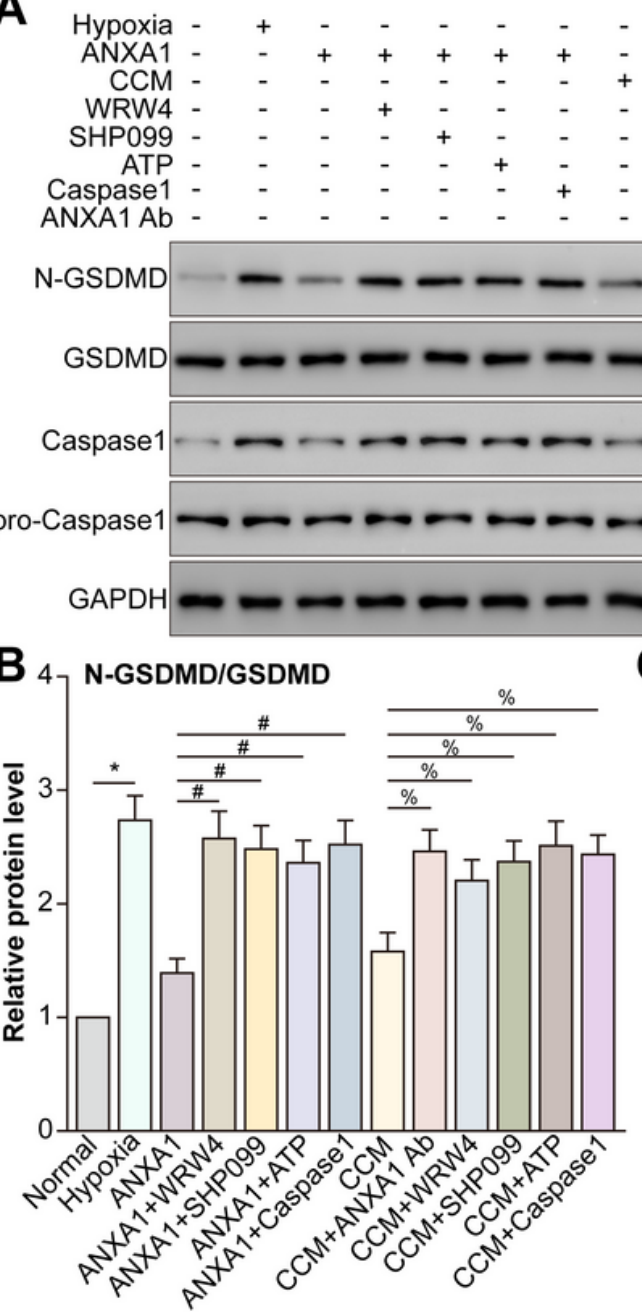

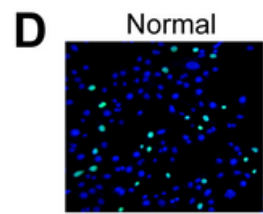

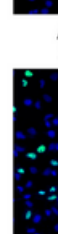

ANXA1+

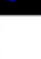

ANXA1+

Hypoxia

ANXA1

ANXA1+WRW4
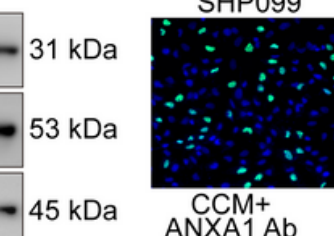

ATP
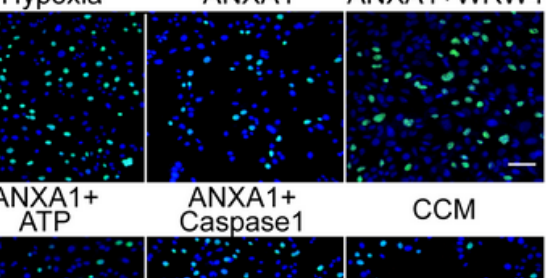
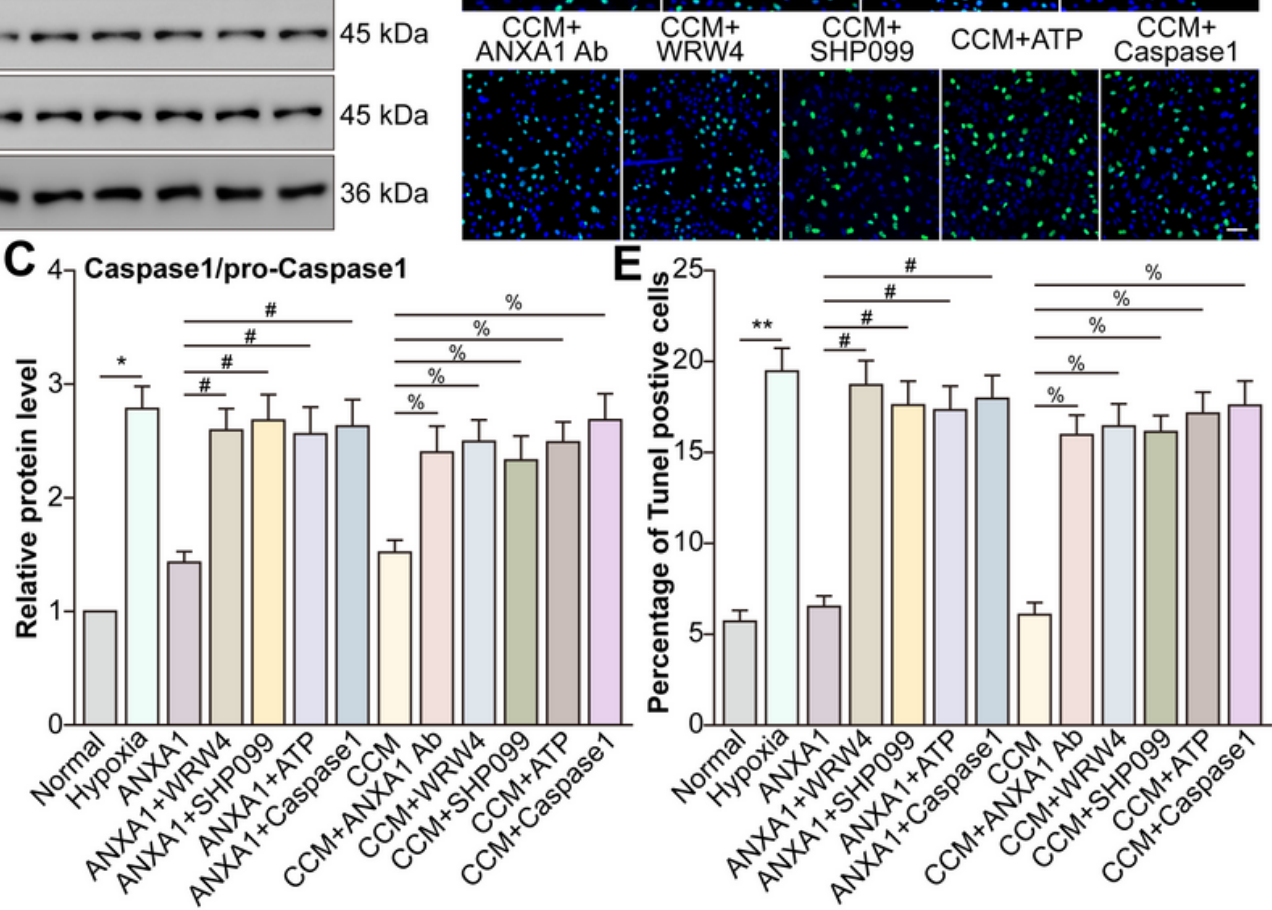

\section{Figure 4}

ANXA1 secreted by ARPE-19 cells inhibits NLRP3 inflammasome-mediated pyroptosis in HCECs by activating the FPR2/SHP2 axis. HCECs were allocated into the normal, hypoxia, human recombinant ANXA1 protein, ANXA1 + WRW4, ANXA1 + SHP099, ANXA1 + ATP, ANXA1 + caspase-1 CRISPR activation plasmid, ARPE-19 cell CCM, CCM + ANXA1 neutralizing antibody, CCM + WRW4, CCM + SHP099, CCM + ATP, and CCM + caspase-1 CRISPR activation plasmid groups. (A) N-GSDMD, GSDMD, caspase-1 and cleaved caspase-1 protein levels in HCECs were measured by Western blot. GAPDH was used as the loading control. (B-C) The mean ratios of N-GSDMD/GSDMD and cleaved caspase-1/caspase-1 were analyzed. ${ }^{*} \mathrm{P}<0.05$ versus the normal group. \#P $<0.05$ versus the ANXA1 group. \% $<0.05$ versus the CCM group. (D) Representative images of TUNEL-stained HCECs are shown. (E) The mean ratio of TUNEL-positive cells was determined. ${ }^{*} \mathrm{P}<0.01$ versus the normal group. \#P $<0.05$ versus the ANXA1 group. \% $\mathrm{P}<0.05$ versus the CCM group. $\mathrm{n}=4$ /each group. 

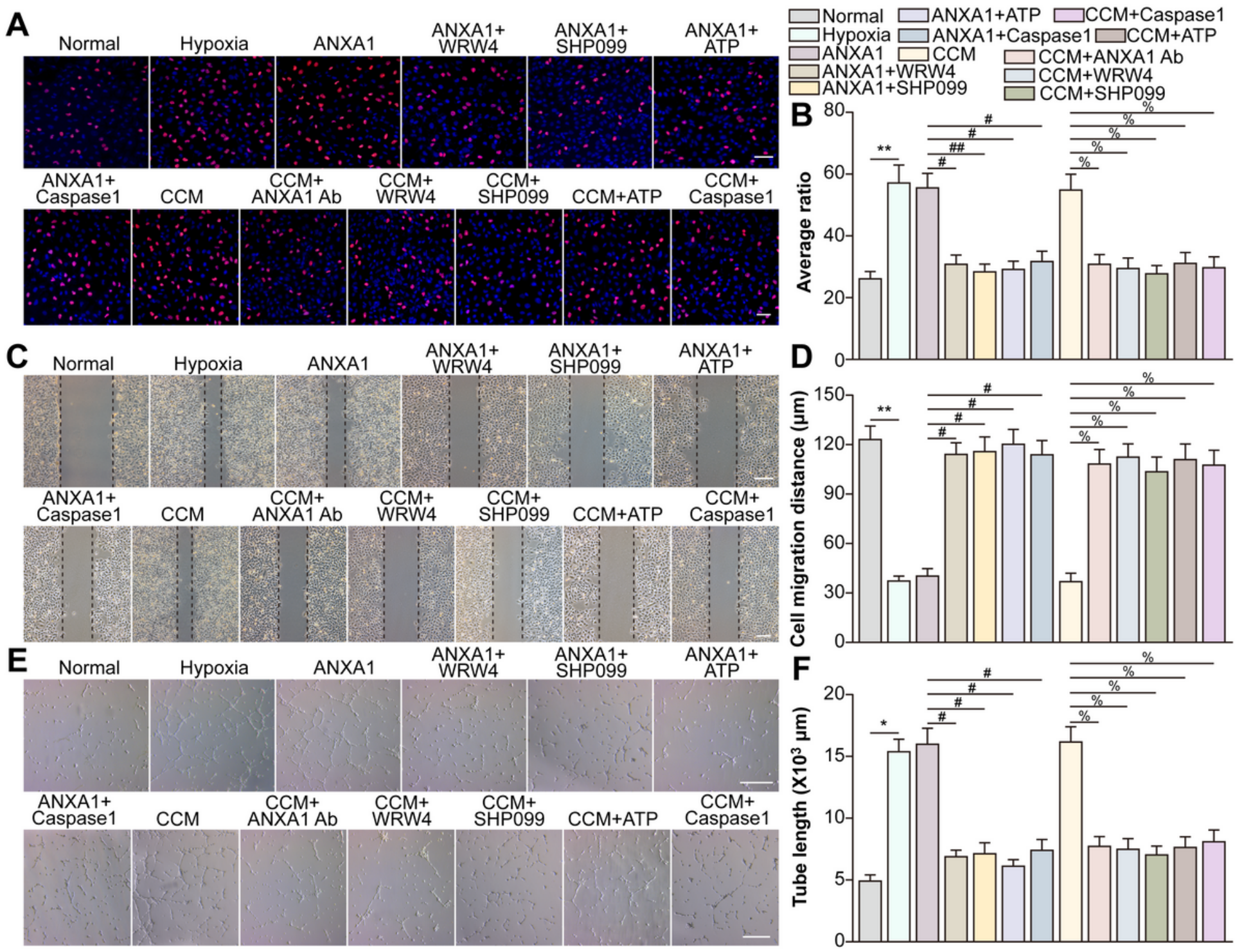

\section{Figure 5}

ANXA1 secreted by ARPE-19 cells promotes the proliferation, migration and tube formation of HCECs by activating the FPR2/SHP2 axis and inhibiting NLRP3 inflammasome-mediated pyroptosis. HCECs were allocated into the normal, hypoxia, human recombinant ANXA1 protein, ANXA1 + WRW4, ANXA1 + SHP099, ANXA1 + ATP, ANXA1 + caspase-1 CRISPR activation plasmid, ARPE-19 cell CCM, CCM + ANXA1 neutralizing antibody, CCM + WRW4, CCM + SHP099, CCM + ATP, and CCM + caspase-1 plasmid groups. (A) The proliferation of HCECs was examined by EdU incorporation assay. Scale bar $=100 \mu \mathrm{m}$. (B) The ratio of EdU-positive cells to DAPI-positive cells was analyzed. (C) The migration of HCECs was examined by wound healing assay. Scale bar $=50 \mu \mathrm{m}$. (D) The migration distance was analyzed. $(E)$ Tube formation assay was used to examine the tube formation capability of HCECs. Scale bar $=200 \mu \mathrm{m}$. (F) The tube length $(\mu \mathrm{m})$ was analyzed. In Fig. $5 \mathrm{~B}, 5 \mathrm{D}$ and $5 \mathrm{~F}$, $* * \mathrm{P}<0.01$ the hypoxia group versus the normal group; $\# P<0.05$ versus the ANXA1 group; \% $<0.05$ versus the CCM group. $n=4$ /each group. 


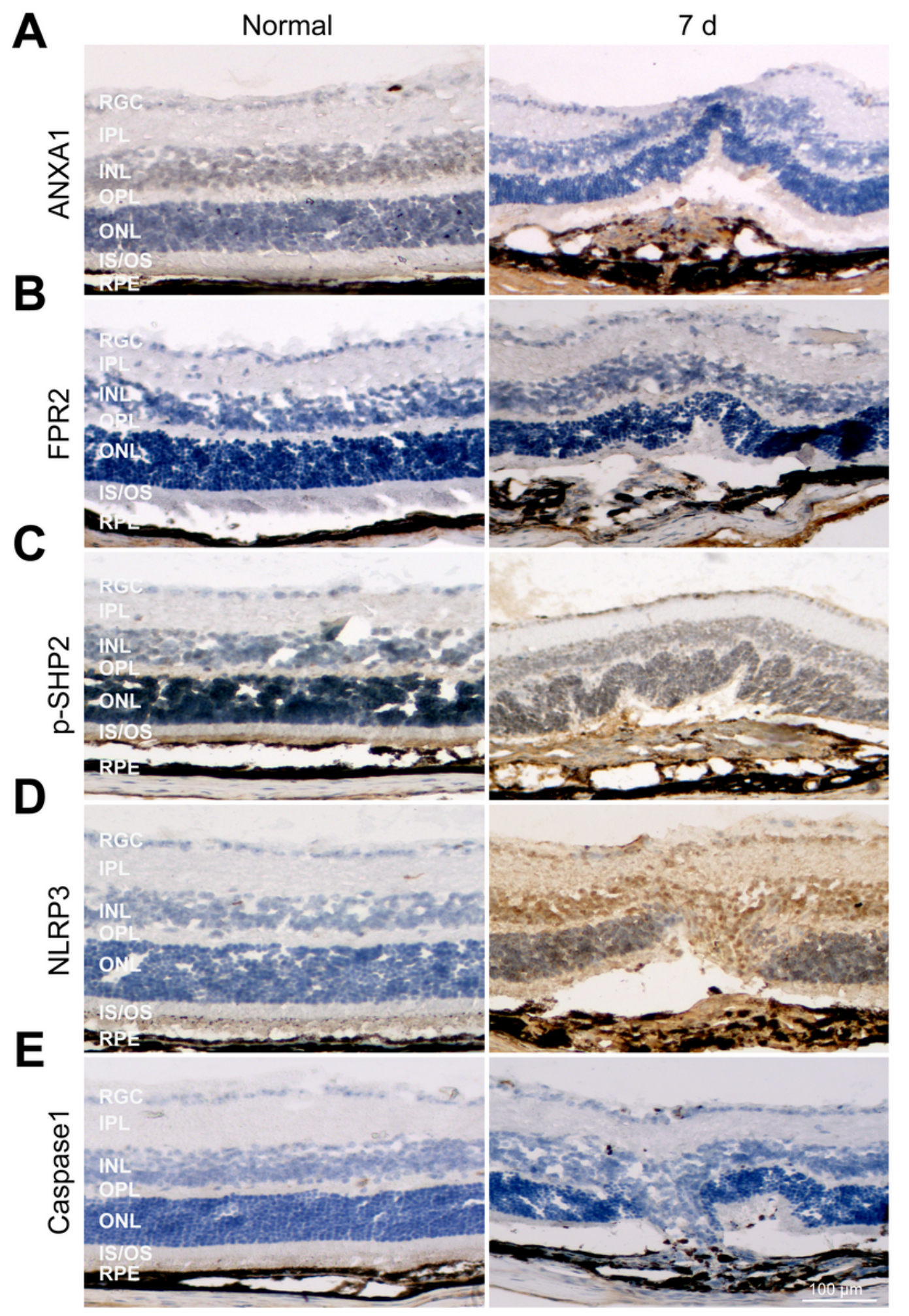

\section{Figure 6}

The ANXA1/FPR2/SHP2/NLRP3 inflammasome/pyroptosis axis is upregulated in laser-induced CNV mice. The mice were allocated into normal and CNV $7 \mathrm{~d}$ groups. (A) ANXA1 was stained on the retinaRPE-choroid complexes. (B) FPR2 was stained on the retina-RPE-choroid complexes. (C) P-SHP2 was stained on the retina-RPE-choroid complexes. (D) NLRP3 was stained on the retina-RPE-choroid complexes. (E) Cleaved caspase-1 was stained on the retina-RPE-choroid complexes. 


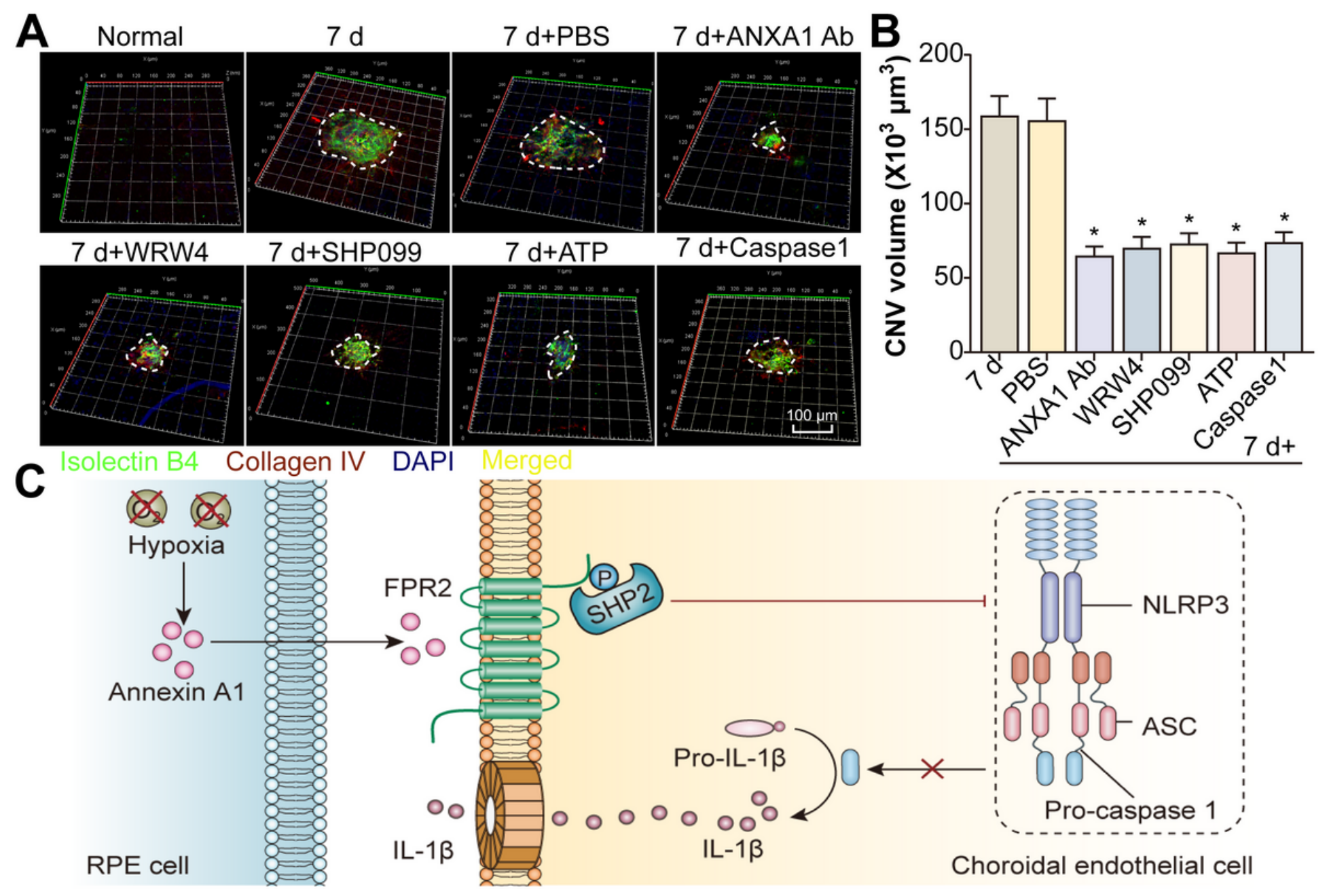

\section{Figure 7}

Inhibition of the ANXA1/FPR2/SHP2/NLRP3 inflammasome/pyroptosis axis decreases the volume of CNV. The mice were allocated into the normal, CNV $7 d$, CNV $7 d+$ PBS, CNV $7 d+$ ANXA1 neutralizing antibody, CNV $7 \mathrm{~d}$ + WRW4, CNV $7 \mathrm{~d}+$ SHP099, CNV $7 \mathrm{~d}+$ ATP, and CNV + caspase-1 plasmid groups. (A) IB4 (green) and collagen IV (red) were analyzed on mouse choroidal flat mounts. (B) The volume of CNV was shown. ${ }^{*} \mathrm{P}<0.05$ versus the CNV $7 \mathrm{~d}$ group. (C) Diagram depicting the mechanisms of the ANXA1/FPR2/SHP2/NLRP3 inflammasome/pyroptosis axis in CNV. After hypoxia, Annexin A1 (ANXA1) secretion from RPE cells increases. ANXA1 binds to FPR2 on the surface of CECs to activate SHP2. SHP2 inhibits the activation of NLRP3 inflammasome and subsequent pyroptosis. Thereafter, proliferation, migration and tube formation of CECs are enhanced. Inhibition of the ANXA1/FPR2/NLRP3 inflammasome/pyroptosis axis mitigates laser-induced CNV in mice. 